\title{
Pharmacological effects of recombinant FGF21 in ovariectomized mice $\mathrm{C} 57 \mathrm{Bl} / 6 \mathrm{~J}$
}

\author{
Antonina Kazantseva \\ ICG SB RAS, Novosibirsk, Russia \\ Nadezhda Kriklivaya \\ ICG SB RAS, Novosibirsk, Russia
}

\author{
Tatyana Yakovleva \\ ICG SB RAS, Novosibirsk, Russia \\ Nadezhda BazhanICG SB RAS, \\ Novosibirsk, Russia
}

\author{
Elena Makarova \\ ICG SB RAS, Novosibirsk, Russia
}

\begin{abstract}
Postmenopausal women have an increased risk of the development of the metabolic syndrome and type 2 diabetes. In pharmacological experiments it was found that hepatic hormone fibroblast growth factor 21 (FGF21) acts as a potent metabolic regulator. But the normalizing metabolic effects of FGF21 have been described only for males. Ovariectomized female mice may be considered as a model of decreased levels of estradiol in women during postmenopause. In this study we investigated the pharmacological effects of recombinant FGF21 in ovariectomized female mice. Ovariectomized animals received daily FGF21 injections ( $1 \mathrm{mkg} / \mathrm{g}$ body weight) for 7 days. On the 7 th day, mice were tested in a glucose tolerance test. Then in mice blood levels of insulin, glucose and adiponectin were determined. Our results showed that FGF21 did not reduce body weight and adiposity, but improved carbohydrate lipid metabolism in estrogen-deficient female mice. These data allow considering FGF21 as a potential regulator for the correction of metabolic syndrome caused by a lack of estrogen in female mice.
\end{abstract}

Keywords - FGF21, ovariectomy, glucose tolerance, female mice

\section{Motivation and Aim}

Postmenopausal women are at increased risk of body weight gain, adiposity, and development of type 2 diabetes. Estradiol usually used for therapy of postmenopausal syndrome. Estradiol treatment improves carbohydrate lipid metabolism, but has a number of negative side effects such as malignant tumors, cardiovascular diseases, and vascular thrombosis. Currently, work is actively underway to discover new pharmacological agents that can normalize metabolic disorders caused by a decrease in estrogens in women after menopause.

Two decades ago, a new hepatic hormone fibroblast growth factor 21 (FGF21) was discovered [1] which acts as a potent metabolic regulator. In pharmacological experiments recombinant FGF21 reduces body weight and significantly improves insulin sensitivity: reduces blood levels of insulin, glucose, free fatty acids, triglycerides, increases glucose tolerance [3]. The normalizing metabolic effects of FGF21 have been described only for males. It is unknown whether FGF21 will inhibit the development of the metabolic syndrome forming in the females due to estrogen deficiency. Ovariectomized female mice may be considered as a model of decreased levels of estradiol in women during postmenopause. Therefore the aim of the study was to investigate the pharmacological effects of FGF21 in ovariectomized female mice.

\section{Methods}

C57BL/6J female mice were kept in the SPF vivarium of the Institute of Cytology and Genetics. All experiments were performed according to the European Convention for the Protection of Vertebrate Animals used for Experimental and other Scientific Purposes (Council of Europe No. 123, Strasbourg 1985) and Russian national instructions for the care and use of laboratory animals. The protocols were approved by the Independent Ethics Committee of the Institute of Cytology and Genetics (Siberian Branch of the Russian Academy of Sciences).

Three experimental groups were formed: sham surgery animals (SHAM) and ovariectomized animals that received injections of solvent (OVX), and ovariectomized animals that received daily recombinant FGF21 injections $(1 \mathrm{mkg} / \mathrm{g}$ body weight) (OVX-FGF21). The injections lasted 7 days. A day 7 after a night of fasting the animals were tested in a glucose tolerance test. The animals were decapitated a day after the last injection.

Blood glucose concentration was determined using the OneTouch Select glucometer. Plasma hormone and metabolites concentrations were determined by using commercial kits according to manufacturer's instruction.

The effect of ovariectomy and FGF21 on the studied parameters in females was determined using a single-factor MANOVA variance analysis (gradations of the factor "experimental group": SHAM, OVX, OVX-FGF21) with multiple comparisons using the post hoc Tukey HSD test.

\section{Results}

Ovariectomy increased body weight and fat mass (Fig. 1A, B). Administration of FGF21 did not cancel this effect of ovariectomy. Ovariectomy did not affect the blood level of insulin and glucose (Fig. 1D, E). The blood level of adiponectin was higher in OVX females than in SHAM females, but the differences did not reach the significance level $(p=0.08)$ (Figure 1F). Ovariectomy reduced glucose tolerance (Fig. 1C). In glucose tolerance test, the area under the curve (AUC) of glucose in the OVX females was higher than in the SHAM females. 


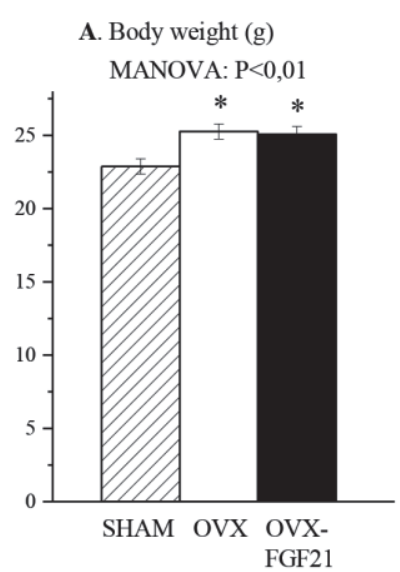

D. Glucose (mmol/l)

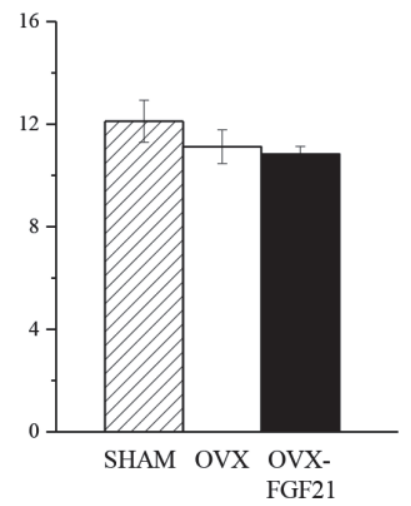

B. Fat mass (g)

MANOVA: $\mathrm{P}<0,01$

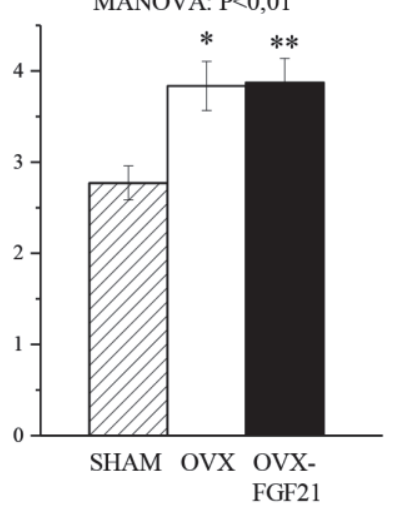

E. Insulin (ng/ml)

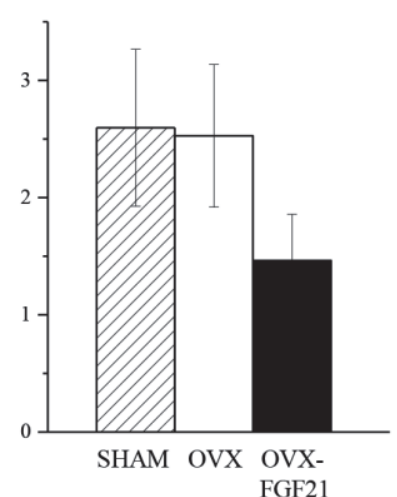

C. AUC

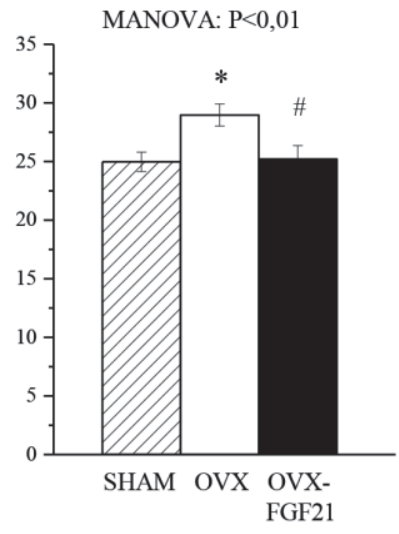

F. Adiponectin (mkg/ml)

MANOVA: $\mathrm{P}<0,01$

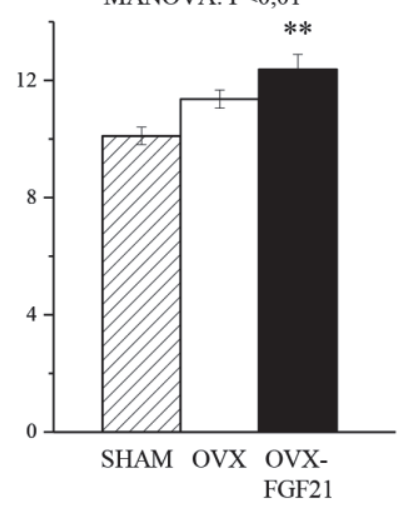

Fig. 1. Body weight (A), fat mass (B), glucose tolerance (C), plasma concentrations of glucose (D), insulin (E) and adiponectin (F) in females of C57Bl/6J mice of three groups: sham surgery animals (hatched column), gonadectomized animals that received injections of solvent (white column), and gonadectomized animals that received injections of recombinant FGF21 ( $1 \mathrm{mkg} / \mathrm{g}$ body weight) (black column).

$* \mathrm{p}<0.05, * * \mathrm{p}<0.01$ compared with SHAM; \# $<<0.05$ compared with OVX (post hoc Tukey HSD test)

In ovariectomized females administration of FGF21 improved carbohydrate lipid metabolism due to effect on the adiponectin blood level and glucose tolerance (Fig. 1C, F). In the OVX-FGF21 females adiponectin blood levels were higher than in the SHAM females and AUC was lower than in the OVX females.

Obviously one of the reasons for the increased glucose tolerance in mice treated with FGF21 is an increase in insulin sensitivity. Adiponectin in known to enhances whole-body insulin sensitivity due to FFA oxidation [2]. An increase in insulin sensitivity in OVX-FGF21 mice is also indicated by the fact that the blood insulin level in the FGF21 females was 2 times lower than in the OVX females, although the differences did not reach the significance level (Fig. 1E).

Thus, our results showed that FGF21 did not reduce body weight and adiposity, but improved carbohydrate lipid metabolism in estrogen-deficient female mice. These data suggests that FGF21 reduced body weight and improved glucose tolerance by two different mechanisms. All together, our results allow considering FGF21 as a potential regulator for the correction of metabolic syndrome caused by a lack of estrogen in female mice.

\section{ACKNOWLEDGMENT}

The study was supported by the Russian Science Foundation, project 17-15-01036. Use of the equipment of the Center for Genetic Resources of Laboratory Animals at ICG SB RAS was supported by the Ministry of Education and Science of Russia (unique project identifier RFMEFI62117X0015).

\section{REFERENCES}

[1] Li et al., "Fibroblast growth factor 21: a novel metabolic regulator from pharmacology to physiology," Front Med, vol. 7(1), pp. 25-30, 2013.

[2] Hojlund et al., "Reduced 540 plasma adiponectin concentrations may contribute to impaired insulin activation of glycogen 541 synthase in skeletal muscle of patients with type 2 diabetes," Diabetologia, vol. 49, pp. 1283-1291, 2006.

[3] Mottillo et al., "FGF21 does not require adipocyte AMP-activated protein kinase (AMPK) or the phosphorylation of acetyl-CoA carboxylase (ACC) to mediate improvements in whole-body glucose homeostasis," Mol Metab, vol. 6(6), pp. 471-481, 2017. 